\title{
WAKE EFFECT ASSESSMENT OF A FLAP TYPE WAVE ENERGY CONVERTER FARM USING A COUPLING METHODOLOGY
}

\author{
Nicolas Tomey-Bozo* \\ Centre for Marine and \\ Renewable Energy (MaREI) \\ University College Cork \\ Ringaskiddy, Ireland \\ Email: nicolas.tomey@ucc.ie \\ Peter Troch \\ Department of Civil Engineering \\ Ghent University \\ Zwijnaarde, Belgium
}

\author{
Jimmy Murphy \\ Centre for Marine and \\ Renewable Energy (MaREI) \\ University College Cork \\ Ringaskiddy, Ireland
}

\author{
Tony Lewis \\ Centre for Marine and \\ Renewable Energy (MaREI) \\ University College Cork \\ Ringaskiddy, Ireland
}

\author{
Aurélien Babarit \\ Ecole Centrale de Nantes \\ CNRS \\ Nantes, France
}

\section{ABSTRACT}

It is expected that large farms of Wave Energy Converters (WECs) will be installed and as part of the consenting process it will be necessary to quantify their impact on the local environment. The objective of this study is to improve the state-ofthe-art of the methodologies to assess the impact a WEC farm has on the incoming wave field through the use of a coupling methodology. A Boundary Element Method (BEM) solver is used to obtain the near-field wave solution accounting for the wavebody interactions within the array of WECs and a Mild-Slope Equation (MSE) model is used to assess the wave transformation in the far-field. The near-field solution obtained from the BEM solver is described as an internal boundary condition in the MSE model and then propagated throughout the domain. The internal boundary condition is described by imposing the solution of the surface elevation in the area where the farm is located. The methodology is applied to flap type WECs that are deployed in shallow water conditions. The validation of the technique is done first for a single flap and then for an array of 5 flaps. Finally, a mild-slope bathymetry and the influence of the changing depth on the wave transformation is assessed in order to prove the versatility of the method to be applied to real scenarios.

\section{NOMENCLATURE}

$\eta$ Surface elevation.

$\Phi$ Velocity potential at the free surface.

$\omega$ Wave frequency.

$g$ Gravitational acceleration.

$C$ Phase velocity.

$C_{g}$ Group velocity.

$k$ Wave number.

$\omega$ Angular frequency.

$\eta^{*} \quad$ Additional surface elevation.

$\Delta x$ grid size in $X$ direction.

$C_{e} \quad$ Energy velocity.

$A_{\omega}$ Incident Wave Amplitude.

$\Gamma$ Excitation moment coefficient.

I Moment of inertia about the $\mathrm{Y}$ axis.

${ }^{*}$ Address all correspondence to this author. 
$A$ Added moment of inertia.

$B$ Radiation damping coefficient.

$H$ Hydrostatic restoring coefficient.

$B_{\text {PTO }}$ PTO damping coefficient.

$\Theta$ Amplitude of motion.

\section{INTRODUCTION}

The presence of Wave Energy Converter (WEC) farms in the ocean will locally affect the wave transformation processes. WECs are designed to absorb part of the incoming wave energy and therefore to reduce the amount of energy density in the leeside of the farm. The quantification of the wake effect generated by a WEC farm will be an important point of the consenting process for the deployment of these technologies. Furthermore, their potential capability to have a sheltering effect on other marine activities taking place on the lee of the farm may open various opportunities. For all these reasons this study aims to improve the state of the art of the methodologies to quantify the wake effect of a WEC farm.

The methodologies employed up to date to quantify the impact of a WEC farm on its surrounding wave field has shown to be missing some features in order to provide a realistic representation of the phenomena. Some studies have used Wave Propagation Models (WPMs) to assess the far-field effect on the leeside of a WEC farm [1-4] by representing intrinsically the devices as absorption cells or source terms. Others have assessed the near-field wave interactions between devices by using Boundary Element Method (BEM) solvers [5,6]. Reference [7] summarises and describes in detail all techniques that have attempted to address this problem. WPMs are accurate solvers of the wave propagation throughout large domains considering realistic conditions such as irregular bathymetries and dissipative processes. However, these models do not accurately represent the local wave-body interactions and rely on external lookup tables describing the absorption capacity of the WECs in order to represent them inherently. BEM solvers are the opposite as they provide accurate solutions of the local wave-body solution by solving the well-known boundary value problem but are limited in terms of the constant depth assumptions and the restricted size domains.

A coupling methodology is applied in this study to fulfil the gap between the near-field results obtained from a BEM solver and the wave propagation in the far-field solved in a WPM. The BEM solver employed is the open-source code NEMOH and the time-dependent Mild-Slope Equation (MSE) model MILDwave is used as WPM. An internal boundary condition is described in the MSE model for each regular wave frequency based on the perturbed wave solution obtained from the BEM solver. The propagation of the perturbed wave is then solved throughout the rest of the domain. The superposition of the perturbed wave and the incident wave computed intrinsically in the MSE model al- lows the computation of the total wave solution. By applying the methodology to a farm of various WECs it enables wave farm wake effects to be assessed.

Previous studies such as $[8,9]$ have employed similar coupling methodologies where the perturbed wave was calculated for point absorbers WECs. The perturbed wave was solved individually for each device and the wave interactions were calculated within the MSE model. In this study a one-step coupling approach is suggested where the perturbed wave is computed for the whole farm with the BEM solver and then imposed as a wave generation surface in the MSE model. This allows for the proper calculation of all wave interactions between devices within the BEM solver before applying the coupling technique. In addition, the technical details of the coupling technique are described by defining the way the internal boundary is set up within the MSE model.

In this paper the numerical tools and the main governing equations are first described. The proposed methodology is then outlined by describing the technical set up of the internal boundary that allows for the coupling between the two solvers. The methodology is first validated for a single WEC case consisting of a flap type device. Then the methodology is validated for the case of a WEC farm composed of 5 flap type devices. The total wave field and relative error plots are then computed for both of these cases. Finally, an additional case showing the versatility of the method when applied to large domains and changing depth bathymetries is outlined.

\section{NUMERICAL TOOLS INVOLVED}

The two hydrodynamic solvers used in this study are described in the following section together with the main governing equations of the problems assessed.

\section{Open-source Boundary Element Method NEMOH}

$\mathrm{NEMOH}$ is an open-source BEM solver developed by Ecole Centrale de Nantes [10] and it is used in this work to obtain the near-field surrounding the WECs. NEMOH is based in the linear potential theory and it calculates the perturbed velocity potential by solving the scattering problem with the appropriate set of boundary conditions as described in [10]. The perturbed velocity potential is obtained as a 3D solution from the well-known linear wave-body interaction boundary value problem. From the potential at the free surface condition $(z=0)$ it is then straightforward to obtain the surface elevation as shown in Eqn. (1).

The scattering problem is divided into one diffraction problem and one radiation problem per degree of freedom for each wave frequency. The diffraction problem is computed considering the body is fixed under the presence of an incoming incident wave. The radiation problem is solved by considering a forced motion of the body in calm conditions (absence of waves). Then 
the total solution of the wave field is obtained as a superposition of the incident wave, and the diffracted and radiated wave obtained from NEMOH. Equation. (2) describes the superposition in terms of surface elevation:

$$
\begin{gathered}
\bar{\eta}(x, y)=\frac{i \omega}{g} \bar{\Phi}(x, y) \\
\bar{\eta}_{t}(x, y)=\bar{\eta}_{i}+\bar{\eta}_{d}+\sum_{n=1}^{6} \bar{\eta}_{r}
\end{gathered}
$$

Over-bar () denotes the complex form of the variable and subscripts $t, i, d$, and $r$ refer to the total, incident, diffracted, and radiated wave respectively.

\section{Mild-slope Equation Model MILDwave}

The wave propagation model MILDwave is used in this study to solve the wave transformation processes throughout large domains and asses the far-field effects. MILDwave is a time-dependent MSE model developed by Ghent University [11] and is part of the phase-resolved type of WPMs. It solves the propagation of surface waves throughout the domain and the interaction with the obstacles (previously defined) by solving the depth-integrated mild-slope equations of Radder and Dingemans [12]. The velocity potential at the free surface and the instantaneous surface elevation are the variables solved for each coordinate of the grid $(\mathrm{x}, \mathrm{y})$ for each instant $t$ of time by the set of differential equations given in Eqn. (3) and (4) bellow.

$$
\begin{gathered}
\frac{\partial \eta}{\partial t}=B \Phi-\nabla(A \nabla \phi) \\
\frac{\partial \Phi}{\partial t}=-g \eta
\end{gathered}
$$

where

$$
\begin{gathered}
B=\frac{\omega^{2}-k^{2} C C_{g}}{g} \\
A=\frac{C C_{g}}{g}
\end{gathered}
$$

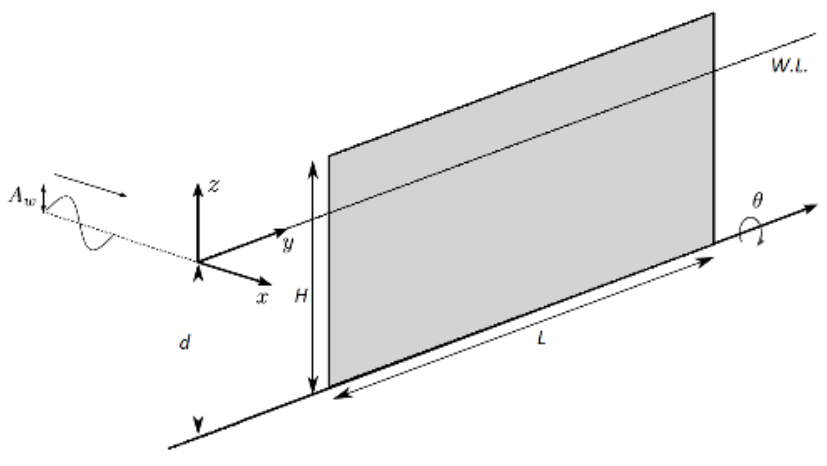

FIGURE 1. FLAP TYPE WEC SKETCH

TABLE 1. MAIN CHARACTERISTICS OF THE FLAP TYPE WEC.

\begin{tabular}{lccc}
\hline Parameter & Coefficient & Value & Units \\
\hline Length & $L$ & 20 & $\mathrm{~m}$ \\
Height & $H$ & 12 & $\mathrm{~m}$ \\
Thickness & $t$ & 1 & $\mathrm{~m}$ \\
Relative density & $\rho_{r}$ & 0.3 & - \\
\hline
\end{tabular}

Incident waves are generated in MILDwave at the offshore boundary by using the source term addition method, i.e. by adding an additional surface elevation $\eta^{*}$ to the calculated value on a wave generation line for each time step given by Eqn. (7) and described in [13]:

$$
\eta^{*}=2 \eta_{i} \frac{C_{e} \Delta t}{\Delta x} \sin \theta
$$

The wave generation line is assumed to be parallel to the $Y$ axis in Eqn. (7).

\section{FLAP TYPE WAVE ENERGY CONVERTER}

The type of WEC considered in this study is a surfacepiercing flap hinged at the bottom of the seabed as shown in Fig. 1. The motion is restricted to pitch therefore only one Degree Of Freedom (DOF) is considered. The shaft about which the flap rotates is at the base of the device. Table 1 shows the main characteristics of the devices.

The amplitudes of angle of motion for each device are calculated based on Eqn. (8). The hydrodynamic coefficients Fe, 
$A$ and $B$, are obtained from the BEM solver where $F e$ represents the excitation force, $A$ the added inertia, and $B$ the radiation damping. The hydrostatic coefficient $H$ and the moment of inertia $I$ are calculated based on the geometry description from Table (1). The Power Take Off (PTO) damping coefficient $B_{\text {PTO }}$ is calculated based on Eqn. (9).

The resultant values of amplitude of motion are used to quantify the radiated wave solution obtained from NEMOH. The surface elevation for the radiated wave is first obtained from the BEM solver in a non-dimensional form relative to a unit of amplitude of motion. In the case of a farm where various WECs are computed within the BEM solver, the terms composing Eqn. (8) are expanded to $n$ dimensions where $n$ represents the number of devices. The expanded form of the equation of motion takes account for all interactions between WECs and therefore determines the amplitude of motion for each device whilst in the presence of the surrounding moving devices. The same procedure is applied when a larger number of DOF are considered. The dimensions of Eqn. (8) are expanded to $n \times j$ where $j$ is the number of DOF of the WEC.

$$
\Theta(\omega)=\frac{A_{\omega} \Gamma(\omega)}{-\omega^{2}(I+A)-i \omega\left(B+B_{P T O}\right)+H}
$$

A passive PTO composed of a damper is used in this study. This configuration was chosen to represent a hydraulic PTO which is usually employed for the flap type WECs. Equation (9) defines the optimum value of the PTO damping coefficient for a specific wave frequency which is theoretically demonstrated in [14]. In the case of an irregular sea state composed of many wave frequencies a fixed value of the PTO damping coefficient was assigned considering the overall statistics of the sea state instead of an optimal value for each frequency that would be constantly changing in time.

$$
B_{P T O}=\sqrt{\left(\frac{H}{\omega}-\omega(I+A)\right)^{2}+B^{2}}
$$

\section{COUPLING METHODOLOGY FOR A SINGLE WEC}

The total wave solution is obtained for each wave frequency from two separate computations. First the incident wave is calculated in MILDwave intrinsically and then the perturbed wave by applying the coupling methodology. A wave generation line is defined at the up-wave boundary for the incident wave computation. Then the incident wave propagates towards the $X$ positive axis across the rest of the empty domain.

For the second computation the perturbed wave is calculated in the BEM solver for the area representing the near-field sur-

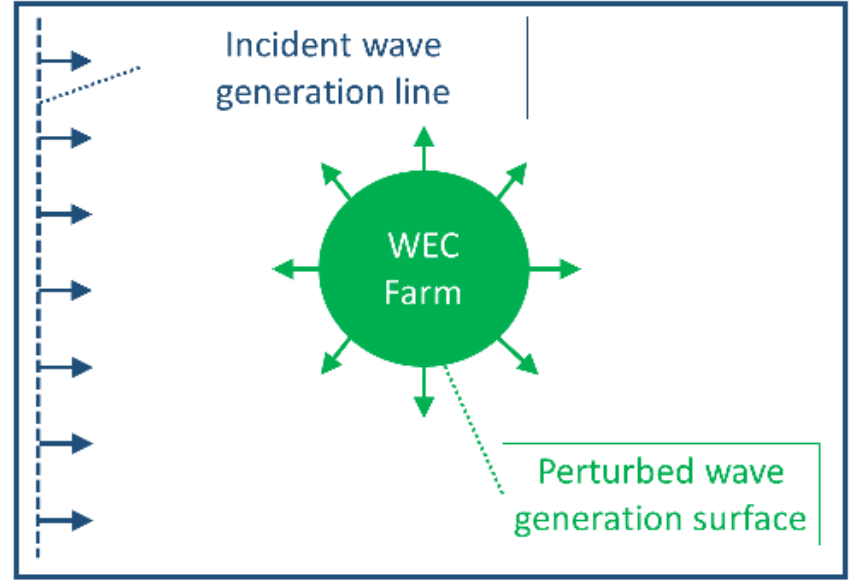

FIGURE 2. METHODOLOGY DESCRIPTION SKETCH

rounding the flap. The perturbed wave is obtained from the superposition of the diffracted wave and radiated wave solution. The perturbed wave representing the near-field is then imposed in MILDwave by forcing the solution of the surface elevation at each instant of time within the same near-field area as an internal boundary condition. The internal boundary acts as a wave generation surface that allows waves to propagate throughout the rest of the domain to obtain the far-field solution. The area size and shape of the internal boundary can be adapted in order to restrict as much as possible the area where the limitations of the BEM are imposed (constant water depths and limited wave transformation processes).

Fig. 2 shows a sketch representation of the methodology with the two different waves generated in MILDwave along the whole domain, the incident wave and the perturbed wave. The way the internal boundary condition is described in this study has been modified and improved as compared to the previous study [15] carried out by the same authors. In [15] the internal boundary was input as a circular wave generation line described in the same manner as an incident wave at the offshore boundary is set up, i.e. by adding an additional surface elevation $\eta^{*}$ on the generation line for each time step. However, the circular wave generation line presents some limitations; the line discretization depends on the radius of the circle, it is limited to circular shapes, and it needs an inner sponge layer that gives reflection problems.

In this study the internal boundary is described by a wave generation surface that can adapt to the desired shape (as far as it surrounds completely the WECs) and does not need a specific parametrisation, i.e. each grid cell contained within the generation surface is attributed its corresponding surface elevation value from the BEM solver solution at each instant of time. 


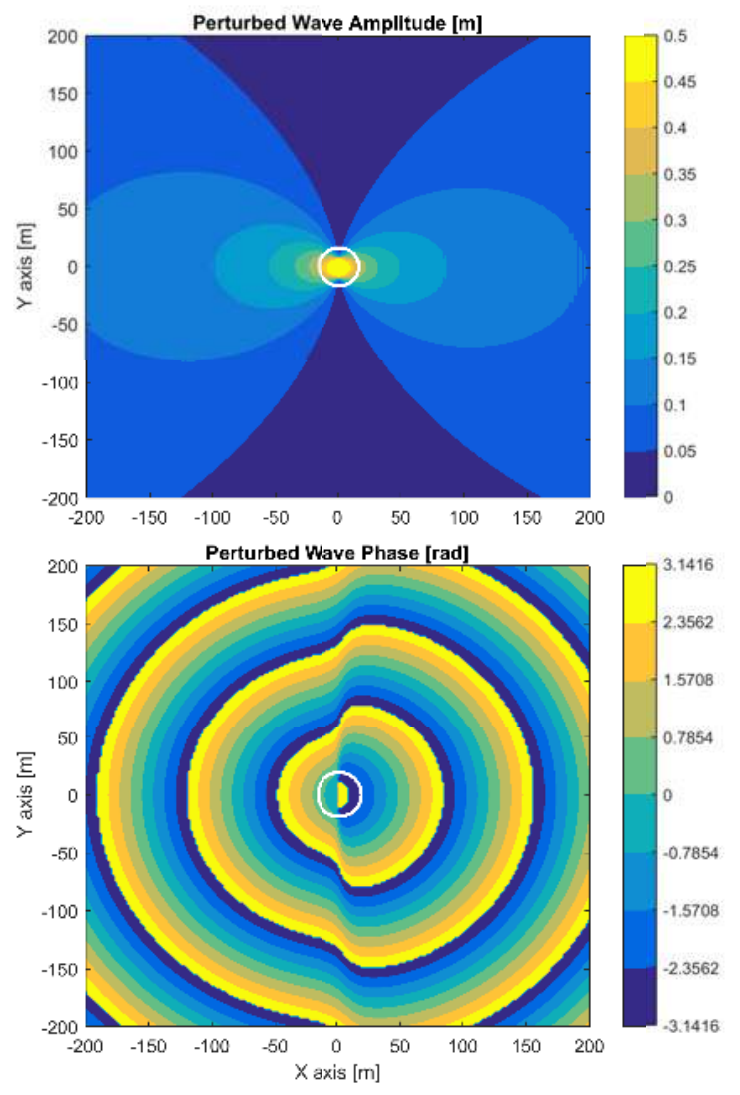

FIGURE 3. PERTURBED WAVE AMPLITUDE AND PHASE FROM NEMOH SOLUTION FOR A SINGLE FLAP

\section{Near-Field From BEM Solver}

The described methodology is first applied to a single WEC case and constant water depth conditions for validation purposes. The perturbed wave surrounding the single flap is obtained in $\mathrm{NEMOH}$ for a domain of $400 \times 400 \mathrm{~m}$. The wave results are plotted in terms of wave amplitude and wave phase in order to facilitate the comparison between the frequency domain results from NEMOH and the time domain results from MILDwave. The wave amplitude and phase correspond to the module and argument of the complex form of the surface elevation $(\bar{\eta})$ respectively. Figure 3 shows the perturbed wave solution across the whole domain corresponding to an incident wave of period of $8 \mathrm{~s}$ and an amplitude of $1 \mathrm{~m}$. A circle surrounding the flap delimits the area corresponding to the near-field that is used in the next stage to describe the wave generation surface in MILDwave. In this case a circular area of radius $R$ equal to $20 \mathrm{~m}$ is used to delimit the near-field.
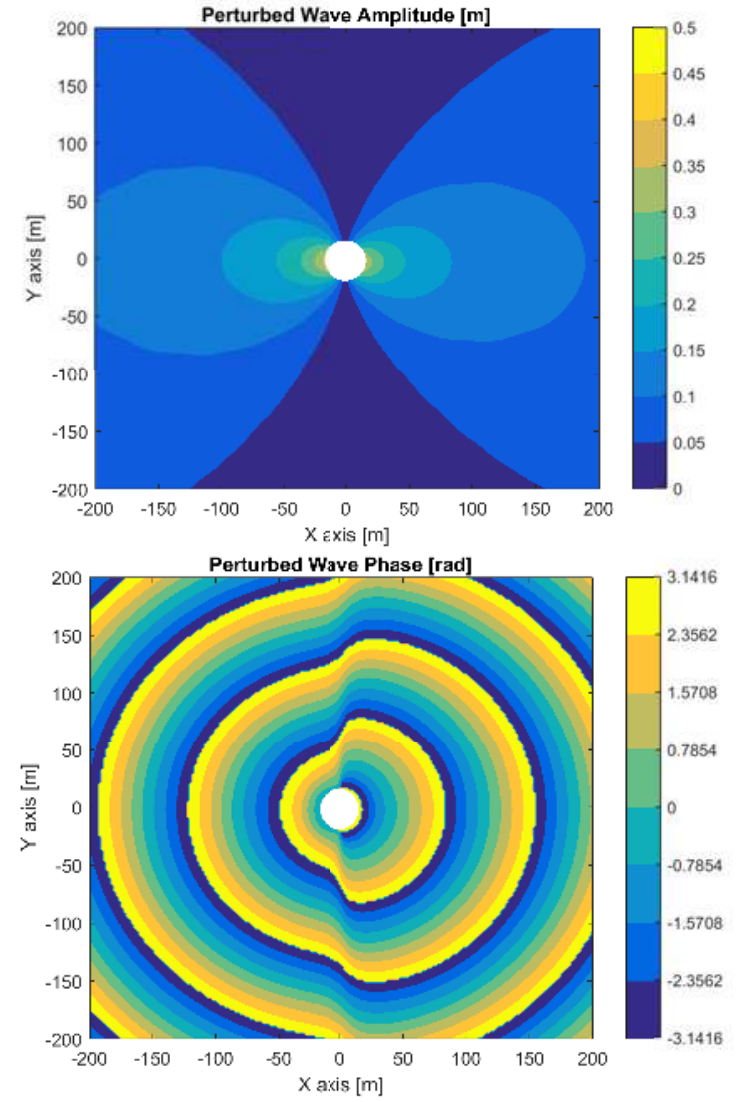

FIGURE 4. PERTURBED WAVE AMPLITUDE AND PHASE FROM MILDWAVE SOLUTION FOR A SINGLE FLAP

\section{Far-Field From Wave Propagation Model}

The near-field solution of the perturbed wave obtained from $\mathrm{NEMOH}$ is now imposed in MILDwave at the same location with respect to the WEC, as a wave generation surface of circular shape with radius $r$ equal to $20 \mathrm{~m}$. The surface elevation solution is imposed in the circular area at each instant of time and propagated throughout the rest of the domain. The same conditions than NEMOH of constant water depths are considered in this case to validate the solution obtained in MILDwave. Figure 4 shows the far-field wave amplitude and phase obtained in MILDwave for the same domain of $400 \times 400 \mathrm{~m}$. The empty disc in the middle of the domain represent the location where the solution is imposed.

Figure 5 shows the percentage error of the perturbed wave solution from MILDwave respect to the solution from NEMOH. The error is calculated based on Eqn. 10 where $A_{M}$ is the wave amplitude result from MILDwave, $A_{N}$ the wave amplitude result from $\mathrm{NEMOH}$, and $\overline{A_{N}}$ the mean value of the wave amplitudes from $\mathrm{NEMOH}$ at the boundary between the wave generation surface and the far-field domain solved in MILDwave. The results 


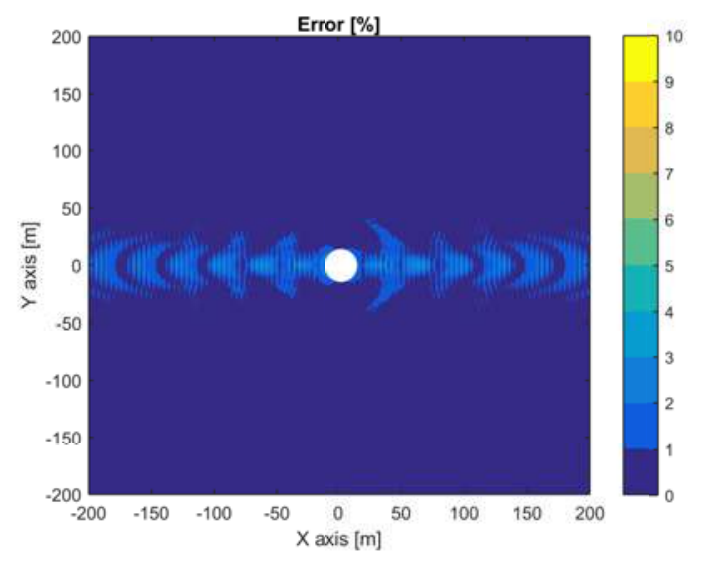

FIGURE 5. PERCENTAGE ERROR BETWEEN MILDWAVE AND NEMOH SOLUTION FOR A SINGLE FLAP

from NEMOH are considered here as a target solution.

$$
\varepsilon(\%)=\frac{A_{N}-A_{M}}{\overline{A_{N}}}
$$

A negligible error is present across the $Y=0$ section with error values remaining below 4\%. Outside this area the error nearly disappears obtaining values that remain under $1 \%$. The error is relatively larger across $Y=0$ section because wave amplitude values are larger across this section. The error remains below acceptable values considering that the maximum percentage error of $4 \%$ corresponds to an absolute error of $0.0036 \mathrm{~m}$, as the average perturbed wave amplitude is equal to $0.09 \mathrm{~m}$.

\section{Total Wave Field}

The total wave amplitude obtained as a result of superposing the perturbed wave from Fig. 4 and the corresponding incident wave is plotted in Fig. 6. The way the internal boundary for the perturbed wave generation is set up (forcing the solution of the wave generation surface) allows us to plot the whole domain including the inner part of the generation surface without any appreciable discontinuity between the near-field and far-field domains.

\section{COUPLING METHODOLOGY FOR A WEC FARM}

The next step was the application of the methodology to a farm of 5 flaps in order to prove the versatility of the technique. The same incident wave conditions and constant water depths as previously outlined are considered. The layout of the farm is defined by a separating spacing between devices of $40 \mathrm{~m}$ in both $X$ and $Y$ directions with an up-wave row composed of 2 flaps and

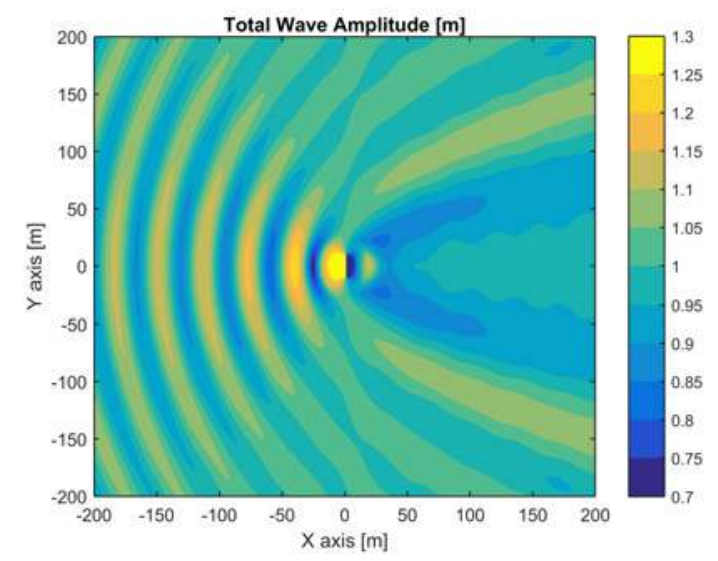

FIGURE 6. TOTAL WAVE AMPLITUDE FROM MILDWAVE SOLUTIONS FOR A SINGLE FLAP

a down-wave row composed of 3 flaps. The flaps from the downwave row are staggered respect to the flaps from the up-wave row.

\section{Near-Field From BEM Solver}

The near-field area surrounding the WEC farm is delimited in this case by a rectangular section of $60 \times 160 \mathrm{~m}(X$ and $Y$ axis respectively). The perturbed wave solution is computed in $\mathrm{NEMOH}$ where all interactions are taken into account. Then the perturbed wave solution corresponding to the rectangular area is described as an internal boundary in MILDwave by means of a wave generation surface. In the case of several WECs the perturbed wave from NEMOH is obtained from the superposition of the diffracted wave and the 5 radiated waves corresponding to each device.

The shape of the wave generation surface is adapted to the shape of the WEC farm. In this case the shape of the near-field is a rectangle that fits the form of the farm by leaving a gap of half of the length of a flap (i.e. $10 \mathrm{~m}$ ). The half flap length distance is left in order to ensure a stable wave generation but the method can be applied with a shorter gap distance. The perturbed wave amplitude and phase obtained from NEMOH are shown in Fig. 7. A rectangle delimits the near-field area which is used to describe the wave generation surface in MILDwave.

\section{Far-Field From Wave Propagation Model}

The perturbed wave is generated now in MILDwave with the wave generation surface of rectangular shape and propagated throughout the rest of the domain. Figure 8 shows the far-field solution obtained in MILDwave in terms of wave amplitude and phase. The same domain size as used in Fig. 7 was chosen in order to compare with the target perturbed wave obtained with 

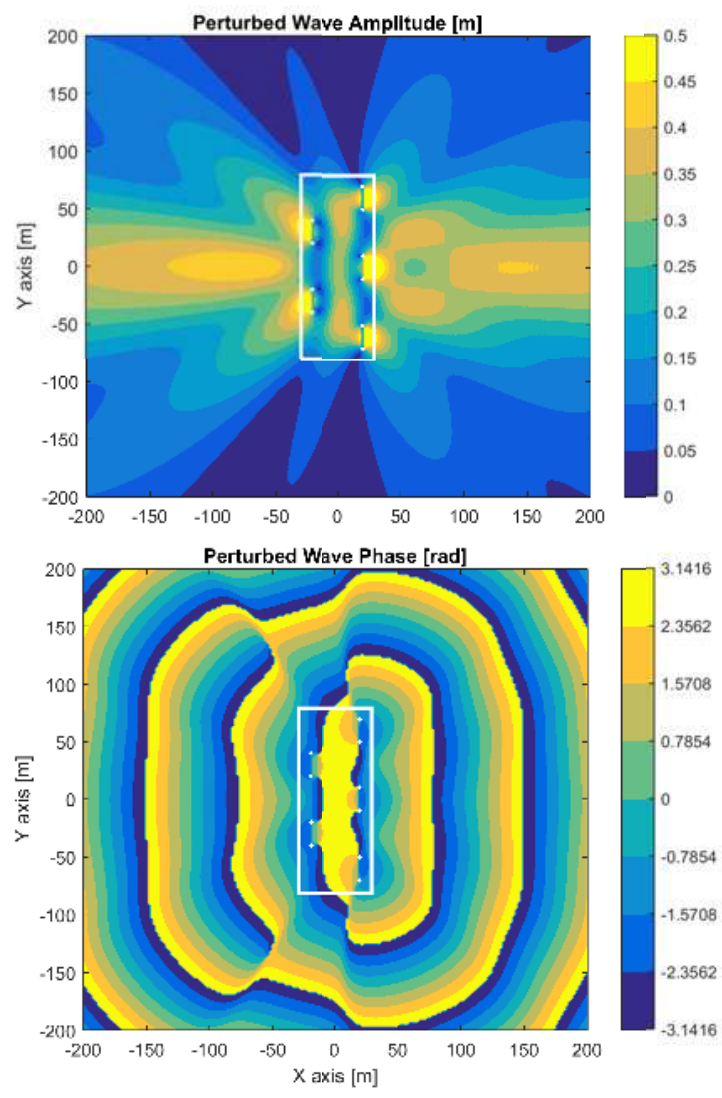

FIGURE 7. PERTURBED WAVE AMPLITUDE AND PHASE FROM NEMOH SOLUTION FOR 5 FLAPS

\section{NEMOH}

The percentage error between the perturbed wave obtained with MILDwave and the perturbed wave obtained with NEMOH is calculated based on Eq. 10 as it is done for the single flap case. Fig. 9 below shows the values obtained for this error.

The maximum error appears along section $Y=0$ where the wave amplitude is the largest of the domain and remains below error values of $2 \%$. This value can be assumed to be negligible considering a percentage error of $2 \%$ corresponds to $0.0055 \mathrm{~m}$ of absolute error, as in this case the mean wave amplitude along the internal boundary is $0.27 \mathrm{~m}$. Therefore, a really good agreement is found for both applications of the coupling technique, the single flap case and the case of the 5 flaps farm.

This application case shows the flexibility of the methodology to adapt the area describing the internal boundary. Whether described by a circular surface or by a rectangular surface, the internal boundary allows the computation of the perturbed wave in MILDwave through a coupling technique. The unique mandatory condition is that the surface used to describe the internal boundary condition surrounds completely the WEC farm in or-

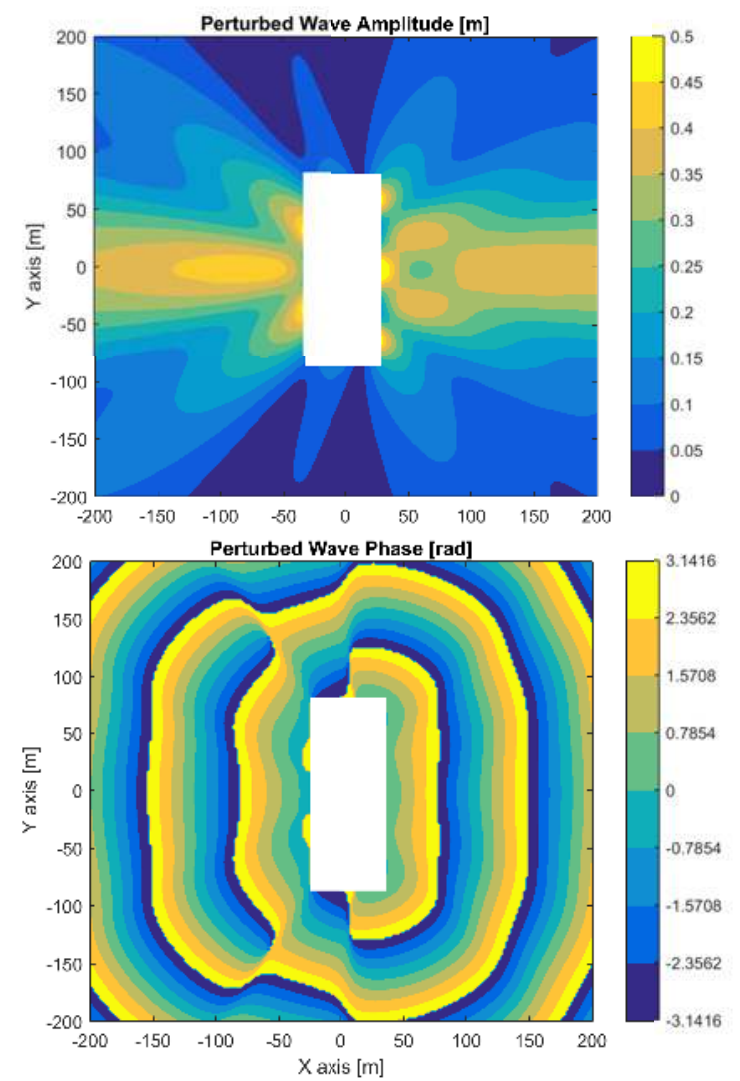

FIGURE 8. PERTURBED WAVE AMPLITUDE AND PHASE FROM MILDWAVE SOLUTION FOR 5 FLAPS

der to describe the correct energy flux.

\section{Total Wave Field}

The total wave field was also obtained for the WEC farm case by superposing the previous results of the perturbed wave to the incident wave computed intrinsically in MILDwave. Figure 10 shows the wave amplitude obtained along the domain for the total wave. As for the single flap case there is no transition in the results between the near-field where the solution of the perturbed wave is imposed from NEMOH and the far-field domain where the solution is solved by the MSE model. The near-field area surrounding the flaps replicates exactly the same values than the ones obtained in NEMOH due to the way the internal boundary is set up.

\section{WAKE EFFECT OF A WEC FARM}

Now that the methodology has been validated for small domains where the comparison was possible against BEM solver solutions, the wake effect of a farm of flaps for a large domain 


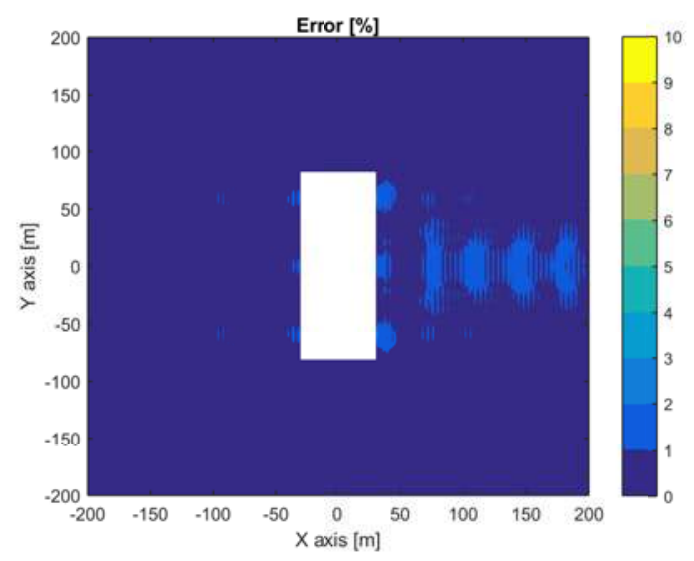

FIGURE 9. PERCENTAGE ERROR BETWEEN MILDWAVE AND NEMOH SOLUTION FOR 5 FLAPS

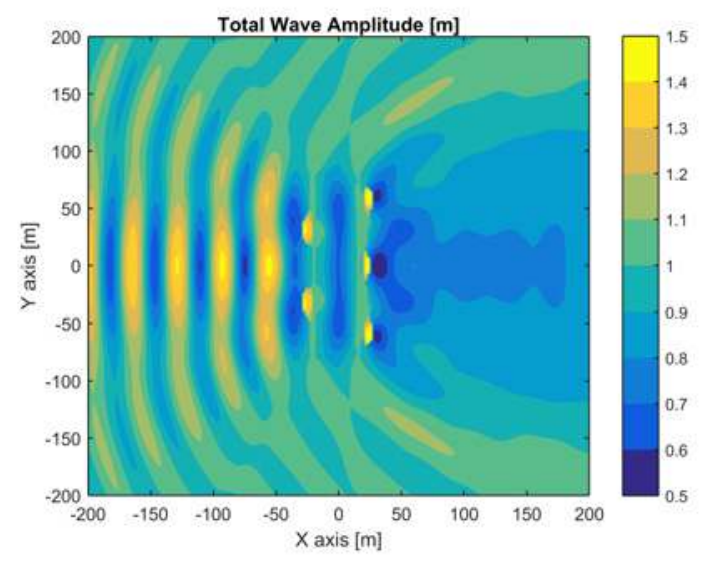

FIGURE 10. TOTAL WAVE AMPLITUDE FROM MILDWAVE SOLUTION FOR 5 FLAPS

is computed in this section. A domain of $1000 \times 2000 \mathrm{~m}(X$ and $Y$ axis respectively) is chosen which gives a broad perspective of the wake effect in the far-field. The wake effect is quantified by the disturbance coefficient $K_{d}$ which in the case of regular waves is obtained by dividing the total wave amplitude (presence of flaps) by the incident wave amplitude (absence of flaps) as described in Eqn. (11).

$$
K_{d}=\frac{A_{t}}{A_{i}}
$$

\section{Wake Effect of a WEC Farm for Constant Water Depths}

First the wake effect is assessed for a constant water depth bathymetry. Figure 11 shows the disturbance coefficient obtained

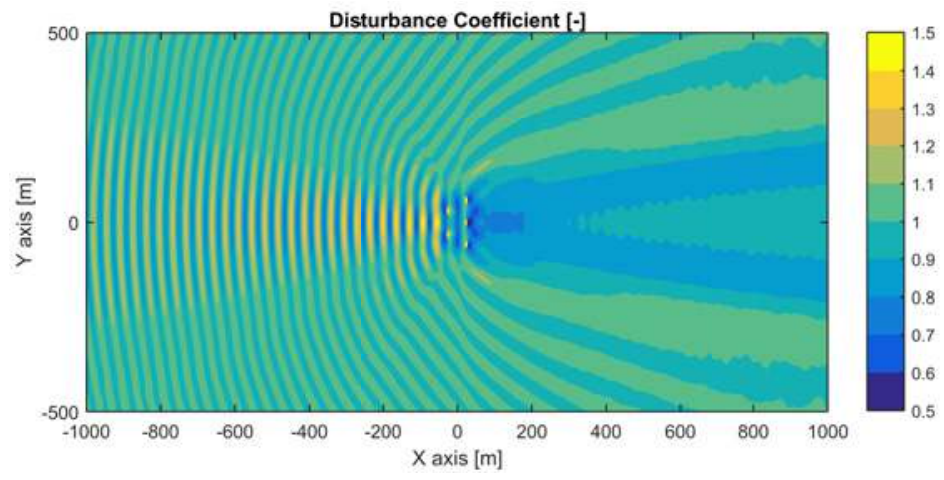

FIGURE 11. DISTURBANCE COEFFICIENT FOR A LARGE DOMAIN WITH CONSTANT WATER DEPTH

along the domain. In the case of constant water depths the incident wave amplitude is equal to 1 therefore the disturbance coefficient is equal to the total wave amplitude.

Clear zones with a reduction of the wave amplitude are found along the plot from Fig. 11. An area of $200 \times 200 \mathrm{~m}$ with values below 0.9 of wave amplitude are found right behind the farm which is equivalent to a $10 \%$ of reduction. Obviously this is a simple case for a small farm of 5 flaps that is used to showcase the method. However with a larger number of flaps a larger zone of wave amplitude reduction can probably be found and potentially protect other marine activities located in the leeside of the WEC farm.

\section{Wake Effect of a WEC Farm for a Mild-Slope Bathymetry}

A case study with a changing depth bathymetry was run next in order to prove the capability of the method to account for irregular bathymetries. The bathymetry is defined by the constant profile along the $Y$ axis shown in Fig. 12. The profile starts with a constant water depths at the up-wave half section and then a mild-slope at the down-wave half section. The mild-slope starts at the centre of the WEC farm $(X=0 \mathrm{~m})$ with 10 meters of water depth and decreases progressively until $5 \mathrm{~m}$ of water depth at $X$ $=1000 \mathrm{~m}$.

Figure 13 shows three plots; first the incident wave amplitude in the absence of flaps, then the total wave amplitude obtained in the presence of flaps, and finally the disturbance coefficient obtained from the division of the two first plots.

Comparing the disturbance coefficient plot from Fig. 13 with Fig. 11 only small differences in the wake effect can be observed. Larger differences may be found in the case of a real bathymetry with irregular profiles in both $X$ and $Y$ directions. 


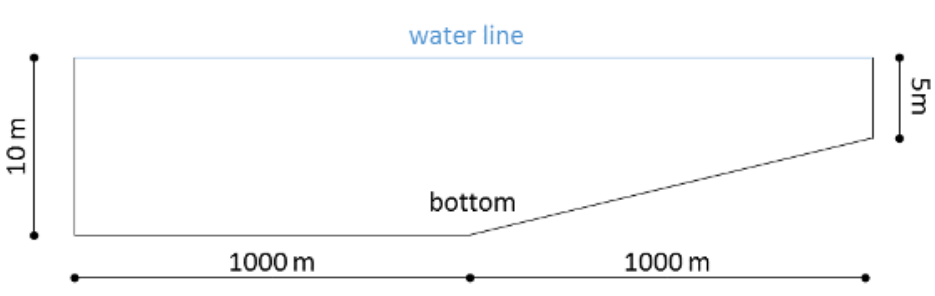

FIGURE 12. BATHYMETRY PROFILE SKETCH

\section{CONCLUSION}

The coupling methodology has shown to give extremely accurate results when comparing the results from the MSE model to the target solutions from the BEM solver. The method has proven to be versatile by tightening the area where the solution of the perturbed wave is imposed to the shape of the farm. This allows the reduction of the area where the limitations of the BEM solvers are assumed.

The methodology remains relatively fast in terms of computational time considering the total calculations for one wave frequency remain less than 10 minutes for the domain size considered in the last section and with a standard computer. The fact of computing all wave interactions within the BEM solver can be demanding in terms of computational time when large WEC farms with many devices are considered. Thus it is the unique way for computing all wave interactions in a farm of WECs within an acceptable computational time. New methodologies are under development to compute wave interactions within an array of WECs based on cylindrical solutions of the perturbed potential from BEM solvers $[6,16]$. These methods can increase significantly the calculation time of the perturbed wave for a large array for constant water depths conditions.

It is straightforward to calculate irregular sea states from the regular wave solutions by superposing all wave components that are present in the considered sea state. This allows the proposed methodology to be used to more accurately quantify the impact of a WEC farm has on the wave climate taking account of realistic conditions, i.e. irregular bathymetries, irregular sea states, and wave transformation processes. The impact on the incident wave climate caused by large WEC farms composed of many devices can be quite significant. Thus it will be mandatory to assess this impact as part of the procedure for the future commissioning of a wave energy farm. In addition, if the assessment finds a WEC farm has a significant sheltering effect, other marine activities sharing the sea space could take advantage of the protected area behind to have calmer water conditions to carry out their tasks.
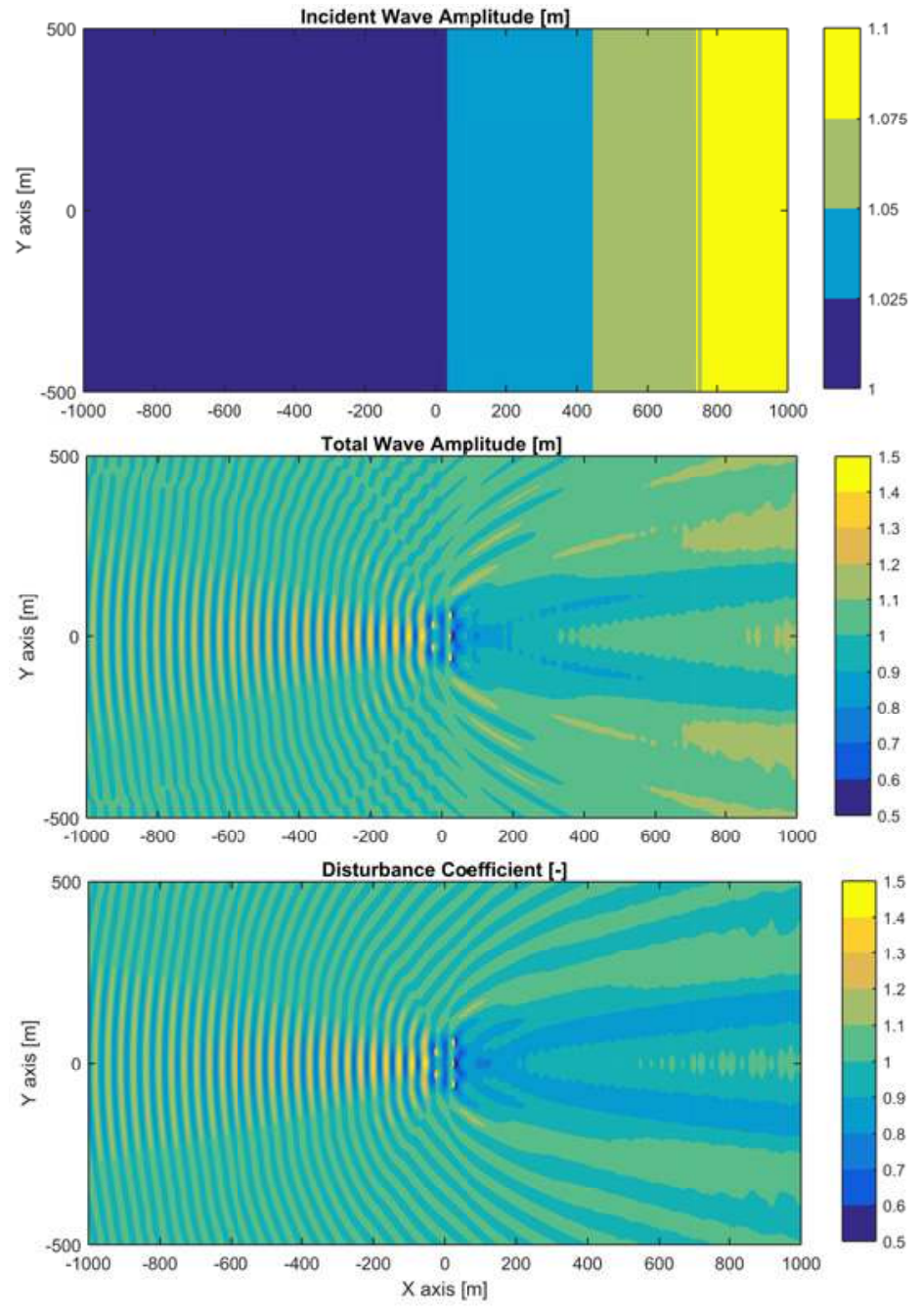

FIGURE 13. INCIDENT WAVE AMPLITUDE, TOTAL WAVE AMPLITUDE, AND DISTURBANCE COEFFICIENT FOR A LARGE DOMAIN WITH A MILD-SLOPE BATHYMETRY

\section{ACKNOWLEDGMENT}

The research leading to these results is part of the OceaNET project, which has received funding from the European Union's Seventh Framework Programme for research, technological development and demonstration under grant agreement no 607656.

\section{REFERENCES}

[1] Beels, C., Troch, P., De Backer, G., Vantorre, M., De Rouck, J.: Numerical implementation and sensitivity analysis of a wave energy converter in a time-dependent mildslope equation model, Coast. Eng., 2010, 57, (5), pp. 471492.

[2] Beels, C., Troch, P., De Visch, K., Kofoed, J.P., De Backer, 
G.: Application of the time-dependent mild-slope equations for the simulation of wake effects in the lee of a farm of Wave Dragon wave energy converters, Renew. Energy, 2010, 35, (8), pp. 1644-1661.

[3] Smith, H.C.M., Pearce, C., Millar, D.L.: Further analysis of change in nearshore wave climate due to an offshore wave farm: An enhanced case study for the Wave Hub site, Renew. Energy, 2012, 40, (1), pp. 51-64.

[4] Astariz, S., Abanades, J., Perez-Collazo, C., Iglesias, G.: Improving wind farm accessibility for operation \& maintenance through a co-located wave farm: Influence of layout and wave climate, Energy Convers. Manag., 2015, 95, pp. 229-241.

[5] Borgarino, B., Babarit, a., Ferrant, P.: Impact of wave interactions effects on energy absorption in large arrays of wave energy converters, Ocean Eng., 2012, 41, pp. 79-88.

[6] Mcnatt, J.C., Venugopal, V., Forehand, D.: A novel method for deriving the diffraction transfer matrix and its application to multi-body interactions in water waves, Ocean Eng., 2014, 94, pp. 173-185.

[7] Folley, M., Babarit, A., Child, B., et al.: A Review of Numerical Modelling of Wave Energy Converter Arrays, in Proc. 31st Int.Conf. on Ocean, Offshore and Arctic Engineering, Rio de Janeiro, Brazil, July 2012, pp. 535-545

[8] Babarit, A., Folley, M., Charrayre, F., Peyrard, C., Benoit, M.: On the modelling of WECs in wave models using far field coefficients,Eur. Wave Tidal Energy Conf. EWTEC, 2013.

[9] Charrayre, F., Peyrard, C., Benoit, M., Babarit, A.: A coupled methodology forwave-body interactions at the scale of a farm of wave energy converters including irregular bathymetry, Proceedings of the ASME 2014 33rd International Conference on Ocean, Offshore and Arctic Engineering, San Francisco, USA, June 2014.

[10] Babarit, A., Delhommeau, G.: Theoretical and numerical aspects of the open source BEM solver NEMOH, Proc. 11th Eur. Wave Tidal Energy Conf., 2015, (September 2015), pp. $1-12$.

[11] Troch, P. 1998. MILDwave A numerical model for propagation and transformation of linear water waves, Internal Report, Department of Civil Engineering, Ghent University. PMid. 9689969.

[12] Radder, A.C., Dingemans, M.W.: Canonical equations gravity waves, weakly nonlinear gravity wavesWave Motion, 1985, 7, pp. 473485.

[13] Lee, C., Suh, K.D.: Internal generation of waves for timedependent mild-slope equations, Coast. Eng., 1998, 34, (12), pp. 35-57.

[14] Zhao, H. tao, Sun, Z. lin, Hao, C. ling, Shen, J. fa: Numerical modelling on hydrodynamic performance of a bottomhinged flap wave energy converter, China Ocean Eng., 2013, 27, (1), pp. 7386.
[15] Tomey-Bozo, N., Murphy, J., Troch, P., Babarit, A., Lewis, T., Thomas, G.: The modelling of a flap type wave energy converter in a time-dependent mild-slope equation model, Renewable Energies Offshore (2016), pp. 277284.

[16] Fabregas Flavia, F., McNatt, C., Rongere F., Babarit, A., Clement A.H. Computation of the Diffraction Transfer Matrix and the Radiation Characteristics in the open-source BEM code NEMOH, Proc. 35th International Conference on Ocean, Offshore and Arctic Engineering, Busan, South Korea, June 2016. 